\title{
Analysis and Design of Two Stage Mismatch Quantizer for Laplacian Source
}

\author{
Zoran H. Peric ${ }^{1}$, Nikola Simic ${ }^{1}$, Milan S. Savic ${ }^{2}$ \\ ${ }^{1}$ Department of Telecommunications, Faculty of Electronic Engineering, University of Nis, \\ Aleksandra Medvedeva 14, 18000 Nis, Serbia \\ ${ }^{2}$ Mathematical Institute of Serbian Academy of Sciences and Arts, \\ Kneza Mihaila 36, 11000 Belgrade, Serbia \\ zoran.peric@elfak.ni.ac.rs
}

\begin{abstract}
${ }^{1}$ Abstract-Two stage quantization is a well-known but still very popular model for signal processing. However, in a number of occasions we have information about a discrete entrance and we do not know the nature of the continuous signal which preceded it. Hence, information source is commonly modelled by using Laplacian or Gaussian distribution but designed quantizers often do not match entire signal range. A typical analysis for discretized input signal does not consider the changes of the continuous signal variance. The aims of this paper are providing an improved analysis by introducing a novel measure CDSVR, designing the second stage quantizer, as well as estimating system performance for mismatched variances. This way, we discuss the influence of A/D conversion on the signal variance and propose an improved model for performance estimation.
\end{abstract}

Index Terms-Mismatch quantization, Modelling, Laplacian source, Two stage quantizer.

\section{INTRODUCTION}

Mismatch quantization has become very popular in recent years and its importance and appliance was researched in a number of papers [1]-[6]. It is generally considered that two stage quantization model is such that first quantizer has a smaller number of quantization levels in comparison to the second quantizer (i.e. required number of bits for transmission is higher for the second quantizer). This way, first quantizer determines the region and the second one determines the level within it [7]-[9]. The kind of two stage quantizer designed in this way is used for standard G. 711 [7]. On the other hand, the model that we discuss consists of two interconnected quantizers and it is performed in [8]. In [8] was concluded that such two stage quantization model should be designed so that second quantizer is described with at least 4 bits lower number of levels in comparison to the first quantizer. However, some systems (e.g. systems for image processing) have to be designed so that second quantizer does not meet this requirement. Here we propose an improved quantization model and performance estimation that will take into account aforementioned problem.

Manuscript received January 05, 2015; accepted March 16, 2015

This research is supported by Serbian Ministry of Education and Sciences (Projects III44006 and TR30245). This research was performed in cooperation with Mathematical institute of Serbian Academy of Sciences and Arts.
In this paper we analyse two interconnected quantizers with a different number of quantization levels. The first quantizer performs analog-to-digital (A/D) conversion with a large number of quantization levels [9], [10]. Its entrance deals with continuous signal that can take any real value from the infinite interval $(-\infty, \infty)$. We decided to choose fixed uniform quantizer for this task, since it has a low complexity but it still provides a high quality of reconstructed signal for high number of quantization levels. Furthermore, the second quantizer performs nonuniform quantization since it provides high quality for additional data compression with a small number of quantization levels. The output signal of the first quantizer represents the input signal at the second quantizer's entrance whereas the input samples at the both first and second quantizer's entrance are modelled with Laplacian distribution.

Let's denote the first $N_{1}$-level quantizer with $Q_{1}$ and second $N_{2}$-level quantizer with $Q_{2}\left(N_{1}>N_{2}\right)$. Quantizer $Q_{2}$ is intended to perform additional compression of previously discretized samples of limited amplitude [11]. In previous studies, we calculated theoretical results based on characteristics of continuous input signal. That way, we have obtained theoretical values of system's performance that have a small deviation in comparison to the experimental results. This study is aimed to determine how changes of signal characteristics, after $\mathrm{A} / \mathrm{D}$ conversion, affect the performance of the whole system.

The paper is organized as follows. In Section II will be described two stage quantization system model - it will be shown design of the both fixed uniform and fixed nonuniform quantizers, as well as measures of the system's performance. Moreover, it will be introduced a novel measure that deals with variances of continuous and discretized signal. Next, numerical and graphical results will be shown in Section III. In the end, conclusions and ideas for future work will be presented in Section IV.

\section{SYSTEM MODEL}

This section's aim is to describe mismatch quantization model by using aforementioned quantizers $\left(Q_{1}\right.$ and $\left.Q_{2}\right)$ and to propose improved modelling that will provide higher accuracy. The improved model deals with a variance of discretized signal instead of continuous variance. 
As it was said above, we discuss Laplacian information source with a memoryless property and a zero mean value

$$
p(x)=\frac{1}{\sqrt{2} \sigma_{x}} \exp \left(-\frac{\sqrt{2}|x|}{\sigma}\right)
$$

where is $\sigma$ standard deviation of the input signal. Primarily we have chosen Laplacian source since it is commonly used in systems for image processing.

Observed system model consists of two stages. In the first stage fixed uniform quantizer $Q_{1}$ converts analog signal to discrete samples, whereas $Q_{2}$ performs additional data compression by using nonuniform quantization in the second stage. Thereby, decision thresholds and representational levels of $Q_{1}$ can be defined with [11]

$$
x_{i}=i \times \Delta=i \times \frac{2 x_{\max }}{N_{1}},
$$

where $i=0, \ldots, N / 2$.

$$
y_{i}=\frac{x_{i-1}+x_{i}}{2}=\frac{2 i-1}{N_{1}} x_{\max },
$$

where $i=1, \ldots, N / 2$. In (2) and (3) $x_{\max }$ denotes the maximal value of continuous signal amplitude which depends on the input signal range. Its optimal values, depending on the number of quantization levels, can be find in [11]. These two expressions define the positive range of fixed uniform quantizer. Since the quantizer is symmetric, in future consideration we will observe just the positive range.

In order to estimate a difference between continuous signal variance of original signal and variance of discretized signal obtained after processing with $Q_{1}$ we introduce a novel measure: continuous - to - discrete - signal - variance ratio $(C D S V R)$. It is defined with

$$
C D S V R=10 \log \frac{\sigma_{x}^{2}}{\sigma_{y}^{2}}[\mathrm{~dB}]
$$

In previous equation $\sigma_{\mathrm{x}}^{2}$ represents the variance of continuous signal whereas $\sigma_{\mathrm{y}}{ }^{2}$ is the variance of discretized signal, obtained after processing with $Q_{1}$. These two parameters can be defined with [9]:

$$
\begin{gathered}
\sigma_{x}^{2}=\sigma_{\text {reff }}^{2} \times 10^{\frac{\sigma_{x}^{2}[\mathrm{~dB}]}{10}}, \\
\sigma_{y}^{2}=2 \sum_{i=1}^{N / 2} y_{i}^{2} \times P_{i},
\end{gathered}
$$

where $\sigma_{\text {reff }}^{2}$ is the referent variance. Furthermore, $P_{\mathrm{i}}$ are probabilities of discrete input levels of the second quantizer

$$
P_{i}=\int_{x_{i}}^{x_{i+1}} p(x) \mathrm{d} x=\frac{1}{2}\left(\exp \left(-\frac{\sqrt{2} x_{i}}{\sigma}\right)-\exp \left(-\frac{\sqrt{2} x_{i+1}}{\sigma}\right)\right),
$$

where is $\sigma=\sigma_{x}$ for calculating $\sigma_{y}^{2}$ in (6).

Design of the nonuniform quantizer $Q_{2}$ is done in two steps. Firstly, we design the optimal compandor with $\mathrm{N}_{2}$ quantization levels for the unit standard deviation $(\sigma=1)$. After that we discuss range variations by introducing the parameter of proportionality $k$. Compandor's compressor function maps the range $(-\infty, \infty)$ to $(-1,1)$ and it can be defined with [12]

$$
c(x)=-1+2 \frac{\int_{-\infty}^{x} p^{1 / 3}(t) \mathrm{d} t}{\int_{-\infty}^{\infty} p^{1 / 3}(t) \mathrm{d} t} .
$$

Its decision thresholds and representational levels obtained in this way are defined with [12]:

$$
\begin{gathered}
t_{i}=\frac{3}{\sqrt{2}} \log \left(\frac{N_{2}}{2\left(N_{2}-i\right)+\left(2 i-N_{2}\right) \exp \left(\frac{-\sqrt{2}}{3} t_{\max }\right)}\right), \\
\omega_{i}=\frac{3}{\sqrt{2}} \log \left(\frac{N_{2}}{2\left(N_{2}-i\right)+1+\left(2 i-1-N_{2}\right) \exp \left(\frac{-\sqrt{2}}{3} t_{\max }\right)}\right),
\end{gathered}
$$

where is $N_{2} / 2<i \leq N_{2}$. Since that this type of quantizer is symmetric, it is valid

$$
t_{i}=-t_{N_{2}-i}
$$

where $0<i \leq N_{2} / 2$.

$$
\omega_{j}^{\prime}=-\omega_{N_{2}-j}^{\prime},
$$

where $1<j \leq N_{2} / 2$. In (9)-(10) parameter $t_{\max }$ denotes the maximal amplitude of the optimal companding quantizer for the unit variance and its values, depending on the number of quantization levels, can be find in [12]. Finally, decision thresholds and representational levels of nonuniform quantizer $Q_{2}$ are

$$
t_{i}=t_{i}^{\prime} \times k
$$

where $N_{2} \leq i \leq N_{2} / 2$.

$$
\omega_{j}=\omega_{j} \times k,
$$

where $1 \leq j \leq N_{2}$. Since the compression process performed in this way enters some information lost, we measure granular and overload distortion that can be defined with [13]-[15]:

$$
D_{g}=2 \sum_{i=N_{2} / 2}^{N_{2}} \sum_{j=1}^{r_{i}}\left(y_{i j}-\omega_{i}\right)^{2} P\left(y_{i j}\right),
$$


$D_{0}=2 \sum_{j=N_{2}-s}^{N_{2}}\left(y_{j}-\omega_{\max }\right)^{2} P\left(y_{j}\right)+2 \int_{x_{\max }}^{\infty}\left(x-\omega_{\max }\right)^{2} p(x) \mathrm{d} x$.

In (15) parameter $r_{i}$ denotes the number of input levels mapped with $\omega_{i}$ whereas $y_{\mathrm{ij}}$ are output levels of $Q_{1}$. Furthermore, $\omega_{\max }=\omega_{N_{2}}$ in (16) whereas parameter $s$ represents the number of output levels from $Q_{1}$ that are not within designed input range of $Q_{2}$.

In the end, total distortion is equal to

$$
D_{t}=D_{g}+D_{o}
$$

Beside CDSVR, overall system performance will be measured by using $S Q N R$ that represents a common measure of performance [12], [14]

$$
\operatorname{SQNR}\left(\sigma^{2}\right)=10 \log _{10}\left(\frac{\sigma^{2}}{D_{t}}\right)[\mathrm{dB}] .
$$

Numerical results will be calculated for the standard model $\left(\operatorname{SQNR}\left(\sigma_{\mathrm{x}}^{2}\right)\right)$ as well as for the proposed model $\left(\operatorname{SQNR}\left(\sigma_{\mathrm{y}}^{2}\right)\right)$

\section{NUMERICAL RESULTS}

In Fig. 1 is shown $C D S V R$ in function of $\sigma_{\mathrm{x}}{ }^{2}$. Observing Fig. 1 we can conclude that values of $\sigma_{y}{ }^{2}$ do not match corresponding values of $\sigma_{\mathrm{x}}{ }^{2}$. Their difference, or in another words a CDSVR value, increases with increasing the continuous variance of input signal $\sigma_{\mathrm{x}}{ }^{2}$. This means that our quantizer is a kind of a mismatch quantizer, since our signal's variance is varying. As a result, design of the second quantizer is not appropriate (it is designed for the unit standard deviation).

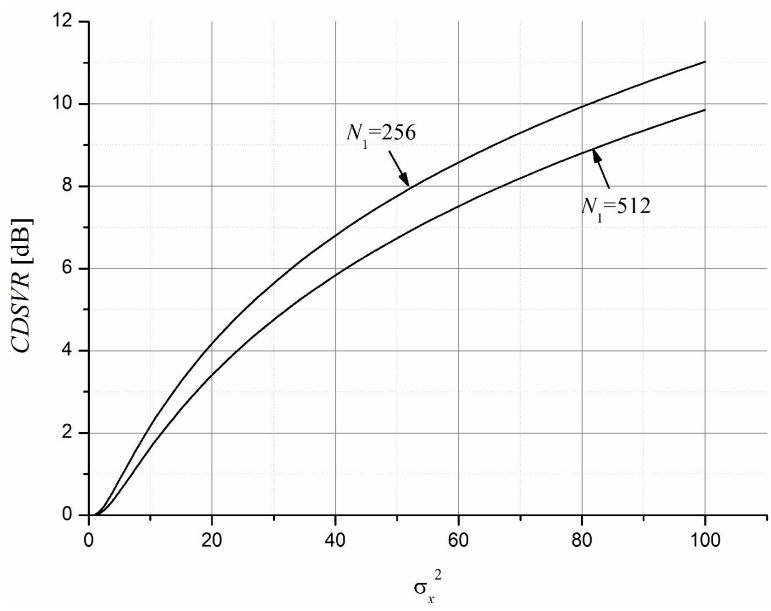

Fig. 1. Continuous-to-discrete-signal-variance ratio (CDSVR).

However, a CDSVR value is lower for a higher number of representational levels $N_{1}$, that can be regarded as a reducing the loss of information occurred due to $\mathrm{A} / \mathrm{D}$ conversion using $Q_{1}$. Consequently, we can expect that proposed modelling that uses $\sigma_{\mathrm{y}}{ }^{2}$ will achieve higher SQNR $\left(\operatorname{SQNR}\left(\sigma_{\mathrm{y}}^{2}\right)\right)$, as it was confirmed experimentally [15].

In Fig. 2 and Fig. 3 the overall SQNR depending on various values of $N_{1}$ in function of $\sigma_{\mathrm{x}}{ }^{2}$ is shown. $S Q N R$ value is calculated in two ways - using the continuous variance of the input signal $\sigma_{\mathrm{x}}{ }^{2}\left(\operatorname{SQNR}\left(\sigma_{\mathrm{x}}^{2}\right)\right)$ and using the variance of discretized signal $\sigma_{\mathrm{y}}{ }^{2}\left(\operatorname{SQNR}\left(\sigma_{\mathrm{y}}^{2}\right)\right)$.

It can be noticed that system's performance calculated in both ways have good matching in the range of small variances $\left(\sigma_{\mathrm{x}}^{2}<5[\mathrm{~dB}]\right)$. With increasing the variance $\sigma_{\mathrm{x}}{ }^{2}$ there is a larger difference of calculated values of $S Q N R$ and system shows better performance in the case when we take into consideration information loss occurred due to $\mathrm{A} / \mathrm{D}$ conversion while processing with $Q_{1}\left(\operatorname{SQNR}\left(\sigma_{\mathrm{y}}{ }^{2}\right)\right)$.

In Fig. 4 and Fig. $5 S Q N R$ depending on parameter of proportionality $k$ for fixed $N_{1}$ and various numbers of representational levels $N_{2}$ is shown.

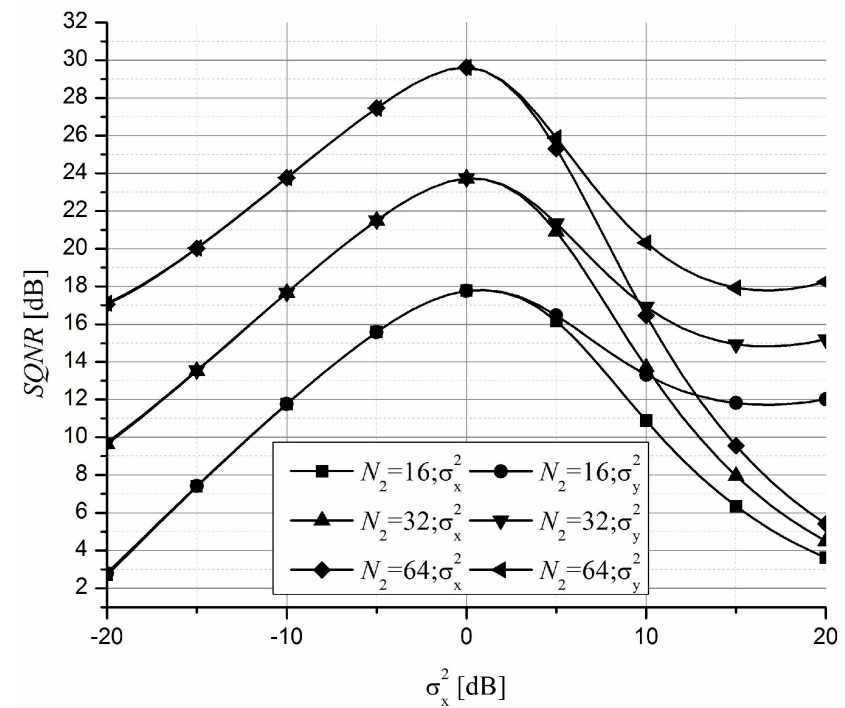

Fig. 2. $S Q N R$ for $N_{1}=256$ and various representational levels of the second quantizer.

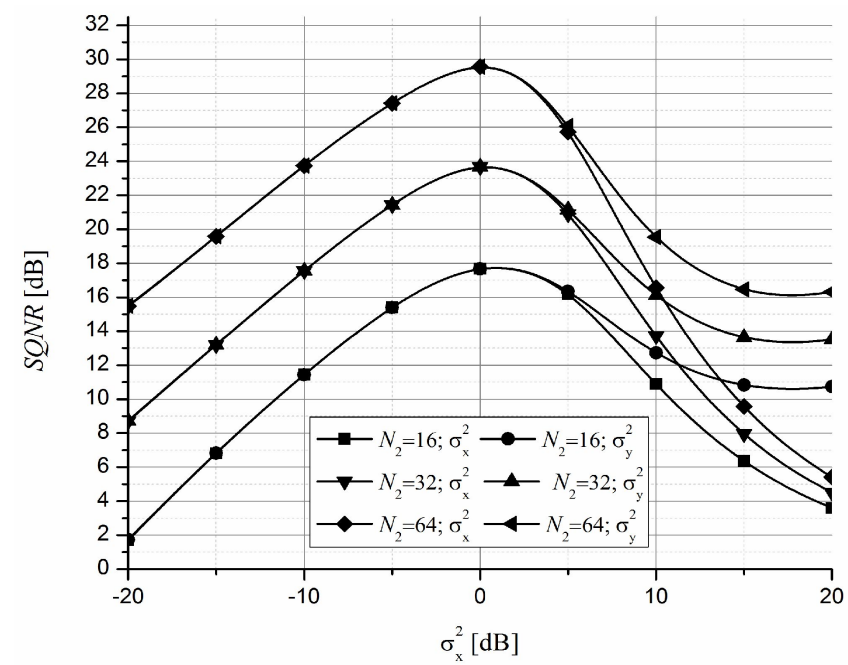

Fig. 3. SQNR for $N_{1}=512$ and various representational levels of the second quantizer.

It can be seen that peaks of the curves are shifted left but their values remain approximately the same by changing the value of parameter $k$. In the range of small variances it can be noticed that $S Q N R$ is slower increasing by incrementing the value of parameter $k$ (for the same $\sigma_{\mathrm{x}}^{2}$ it is obtained higher $S Q N R$ for lower $k$ ).

On the other hand, for $\sigma_{\mathrm{x}}^{2}>5[\mathrm{~dB}], S Q N R$ value decreases by decreasing parameter $k$. Changing this parameter affects the both $\operatorname{SQNR}\left(\sigma_{\mathrm{x}}^{2}\right)$ and $\operatorname{SQNR}\left(\sigma_{\mathrm{y}}^{2}\right)$ in the same way. 


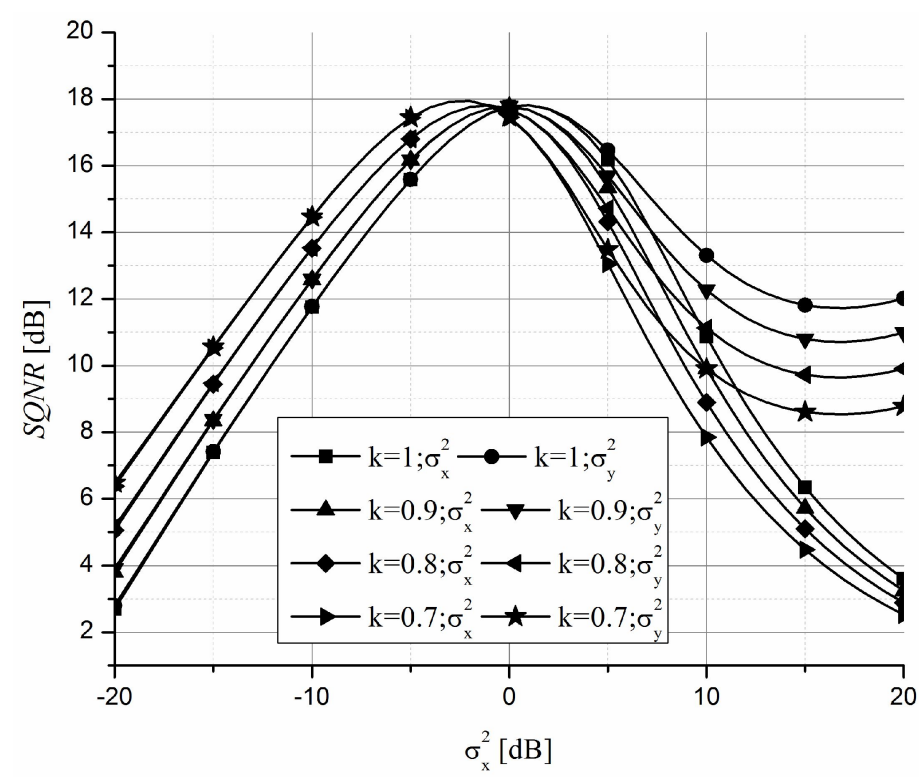

Fig. 4. $S Q N R$ for various values of parameter $k\left(N_{1}=256 ; N_{2}=16\right)$.

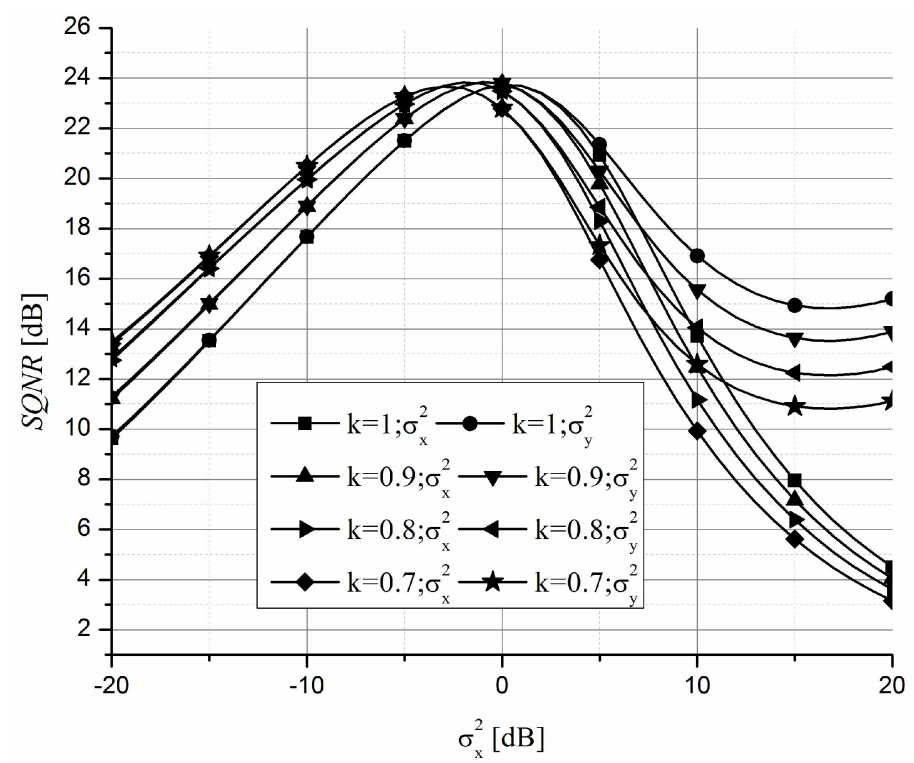

Fig. 5. $S Q N R$ for various values of parameter $k\left(N_{1}=256 ; N_{2}=32\right)$.

In Table I average $\operatorname{SQNR}\left(\operatorname{SQNR}\left(\sigma_{\mathrm{x}}^{2}\right)\right.$ and $\left.\operatorname{SQNR}\left(\sigma_{\mathrm{y}}^{2}\right)\right)$ for various values of parameter $k$ is shown.

TABLE I. AVERAGE SQNR FOR VARIOUS VALUES OF THE BOTH PARAMETER $K$ AND $N_{2}\left(N_{1}=256\right)$

\begin{tabular}{|c|c|c|c|c|}
\hline & \multicolumn{2}{|c|}{$N_{2}=16$} & \multicolumn{2}{|c|}{$N_{2}=32$} \\
\hline$k$ & $\begin{array}{c}S Q N R_{\mathrm{av}}\left(\sigma_{\mathrm{x}}^{2}\right) \\
{[\mathrm{dB}]}\end{array}$ & $\begin{array}{c}S Q N R_{\mathrm{av}}\left(\sigma_{\mathrm{y}}^{2}\right) \\
{[\mathrm{dB}]}\end{array}$ & $\begin{array}{c}S Q N R_{\mathrm{av}}\left(\sigma_{\mathrm{x}}^{2}\right) \\
{[\mathrm{dB}]}\end{array}$ & $\begin{array}{c}S Q N R_{\mathrm{av}}\left(\sigma_{\mathrm{y}}^{2}\right) \\
{[\mathrm{dB}]}\end{array}$ \\
\hline 1 & 11.00236 & 12.60121 & 15.60852 & 17.66160 \\
\hline 0.9 & 11.04911 & 12.55164 & 15.74937 & 17.67307 \\
\hline 0.8 & 11.10471 & 12.49266 & 15.72813 & 17.49095 \\
\hline 0.7 & 11.10435 & 12.35924 & 15.37577 & 16.98225 \\
\hline
\end{tabular}

Obtained average values refer to corresponding graphically presented results in Fig. 4 and Fig. 5 for the range $\sigma_{\mathrm{x}}^{2} \in[-20 \mathrm{~dB}, 20 \mathrm{~dB}]$. It can be concluded that in the aforementioned range, varying the parameter $k$ does not have a significant influence on the overall $S Q N R_{\text {av }}$ of the observed system.

Table II shows average SQNR $\left(S Q N R_{a v}\left(\sigma_{\mathrm{x}}^{2}\right)\right.$ and $\left.S Q N R_{a v}\left(\sigma_{\mathrm{y}}^{2}\right)\right)$ for various sub-ranges of input continuous variance $\left(\sigma_{\mathrm{x}}^{2}\right)$ in function of parameter $k$. We can conclude that parameter $k$ affects overall $S Q N R_{a v}$ in all sub-ranges and its impact increases with decreasing the width of the range.

\section{CONCLUSIONS}

In this paper we discuss limitations of two stage quantization modelling that uses just a variance of continuous signal $\left(\sigma_{\mathrm{x}}^{2}\right)$. Moreover, we introduce a new measure of system's performance $(C D S V R)$. Obtained results show that aforementioned modelling does not provide a real estimation of $S Q N R$ for input variances $\sigma_{\mathrm{x}}{ }^{2}>5[\mathrm{~dB}]$. Consequently, modelling should deal with a variance of discretized signal $\left(\sigma_{\mathrm{y}}{ }^{2}\right)$ for higher accuracy. In the end, we observe the impact of the input range choice (i.e. parameter $k$ influence) on the system's performance.

Future work will be focused on applying the model to systems used for image processing. Also, we will research the difference of mismatch and proposed modelling for estimation of peak signal-to-quantization-noise ratio $(P S Q N R)$ and average bit-rate $\left(R_{b}\right)$. 
TABLE II. AVERAGE SQNR FOR DIFFERENT SUB-RANGES OF INPUT SIGNAL VARIANCE IN THE FUNCTION OF PARAMETER $K\left(N_{1}=256\right.$;

\begin{tabular}{|c|c|c|c|c|c|c|}
\hline \multicolumn{7}{|c|}{$\left.N_{2}=32\right)$} \\
\hline & \multicolumn{2}{|c|}{$\sigma_{\mathrm{x}}^{2} \in[-10,20] \mathrm{dB}$} & \multicolumn{2}{|c|}{$\sigma_{x}^{2} \in[-20,10] d B$} & \multicolumn{2}{|c|}{$\sigma_{x}^{2} \in[-10,10] d B$} \\
\hline$k$ & $\operatorname{SQNR}_{\mathrm{av}}\left(\sigma_{\mathrm{x}}^{2}\right)[\mathrm{dB}]$ & $S Q N R_{\mathrm{av}}\left(\sigma_{\mathrm{y}}^{2}\right)[\mathrm{dB}]$ & $S Q N R_{\mathrm{av}}\left(\sigma_{\mathrm{x}}^{2}\right)[\mathrm{dB}]$ & $\operatorname{SQNR}_{\mathrm{av}}\left(\sigma_{\mathrm{y}}^{2}\right)[\mathrm{dB}]$ & $S Q N R_{\mathrm{av}}\left(\sigma_{\mathrm{x}}^{2}\right)[\mathrm{dB}]$ & $S Q N R_{\mathrm{av}}\left(\sigma_{\mathrm{y}}^{2}\right)[\mathrm{dB}]$ \\
\hline 1 & 16.39667 & 19.09979 & 18.09774 & 18.44468 & 20.44655 & 20.94060 \\
\hline 0.9 & 16.11152 & 18.64390 & 18.53039 & 18.88257 & 20.38928 & 20.89165 \\
\hline 0.8 & 15.63079 & 17.95074 & 18.75263 & 19.09579 & 20.04918 & 20.53874 \\
\hline \multirow[t]{3}{*}{0.7} & 14.99498 & 17.10880 & 18.53101 & 18.86237 & 19.47140 & 19.94448 \\
\hline & \multicolumn{2}{|c|}{$\sigma_{\mathrm{x}}^{2} \in[-5,15] \mathrm{dB}$} & \multicolumn{2}{|c|}{$\sigma_{\mathrm{x}}^{2} \in[-15,5] \mathrm{dB}$} & \multicolumn{2}{|c|}{$\sigma_{\mathrm{x}}^{2} \in[-5,5] \mathrm{dB}$} \\
\hline & $S Q N R_{\mathrm{av}}\left(\sigma_{\mathrm{x}}^{2}\right)[\mathrm{dB}]$ & $S Q N R_{\mathrm{av}}\left(\sigma_{\mathrm{y}}^{2}\right)[\mathrm{dB}]$ & $\operatorname{SQNR}_{\mathrm{av}}\left(\sigma_{\mathrm{x}}^{2}\right)[\mathrm{dB}]$ & $\operatorname{SQNR} R_{\mathrm{av}}\left(\sigma_{\mathrm{y}}^{2}\right)[\mathrm{dB}]$ & $S Q N R_{\mathrm{av}}\left(\sigma_{\mathrm{x}}^{2}\right)[\mathrm{dB}]$ & $\operatorname{SQNR}_{\mathrm{av}}\left(\sigma_{\mathrm{y}}^{2}\right)[\mathrm{dB}]$ \\
\hline 1 & 18.25484 & 20.04838 & 20.09190 & 20.13460 & 22.70497 & 22.77518 \\
\hline 0.9 & 17.70619 & 19.42230 & 20.67117 & 20.72220 & 22.68575 & 22.77255 \\
\hline 0.8 & 16.92263 & 18.51965 & 20.98754 & 21.04420 & 22.30115 & 22.39910 \\
\hline 0.7 & 16.00386 & 17.48337 & 20.86813 & 20.93039 & 21.62943 & 21.73864 \\
\hline
\end{tabular}

\section{REFERENCES}

[1] J. Nikolic, Z. Peric, A. Jovanovic, "Variance mismatch analysis of unrestricted polar quantization for Gaussian source", IEEE Signal Processing Letters, vol. 21, no. 5, pp. 540-544. [Online]. Available: http://dx.doi.org/10.1109/LSP.2014.2309093

[2] S. Na, "Asymptotic formulas for mismatched fixed-rate minimum MSE Laplacian quantizers", IEEE Signal Process. Lett., vol. 15, pp. 13-16, 2008. [Online]. Available: http://dx.doi.org/ 10.1109/LSP.2007.910240

[3] S. Na, "Variance-mismatched fixed-rate scalar quantization of Laplacian sources", IEEE Trans. Inf. Theory, vol. 57, no. 7, pp. 4561-4572, 2011. [Online]. Available: http://dx.doi.org/10.1109/ TIT.2011.2146390

[4] S. Na, "Asymptotic formulas for variance-mismatched fixed-rate scalar quantization of a Gaussian source", IEEE Trans. Signal Process., vol. 59, no. 5, pp. 2437-2441, 2011. [Online]. Available: http://dx.doi.org/10.1109/TSP.2011.2112354

[5] S. Na, D. L. Neuhoff, "On the support of MSE-optimal, fixed-rate, scalar quantizers", IEEE Trans. Inf. Theory, vol. 47, no. 7, pp. 2972 2982, 2001. [Online]. Available: http://dx.doi.org/10.1109/18.959274

[6] S. Na, D. L. Neuhoff, "Asymptotic MSE distortion of mismatched uniform scalar quantization", IEEE Trans. Information Theory, vol. 58, no. 5, 2012. [Online]. Available: http://dx.doi.org/10.1109/ TIT.2011.2179843
[7] ITU-T Recommendation G.711.1 Wideband embedded extension for G.711 pulse code modulation, 2008

[8] R. M. Gray, D. L. Neuhoff, "Quantizaton", IEEE Trans. on Information Theory, vol. 44, no. 6, pp. 2325-2383, 1998. [Online]. Available: http://dx.doi.org/10.1109/18.720541

[9] N. S. Jayant, P. Noll, Digital Coding of Waveforms, Prentice Hall Pb, 1984.

[10] Q. Yun, Shi, Huifnag Sun, Image and Video Compression for Multimedia Engineering, Taylor \& Francis Group, 2008.

[11] M. Savic, Z. Peric, M. Dincic, "Design of forward adaptive uniform quantizer for discrete input samples for Laplacian source", Elektronika Ir Elektrotechnika, no. 9, pp. 73-76, 2010.

[12] Z. Peric, M. Petkovic, M. Dincic, "Simple compression algorithm for memoryless Laplacian source based on the optimal companding technique", Informatica, vol. 20, no. 1, pp. 99-114, 2009.

[13] M. Savic, Z. Peric, M. Dincic, "An algorithm for grayscale image compression based on the forward adaptive quantizer designed for signals with discrete amplitudes", Elektronika Ir Elektrotechnika, no. 2, pp. 13-16, 2012. [Online]. Available: http://dx.doi.org/ 10.5755/j01.eee.118.2.1166

[14] M. Savic, Z. Peric, M. Dincic, "Coding algorithm for grayscale images based on piecewise uniform quantizers", Informatica, vol. 23, no. 1, pp. 125-140, 2012.

[15] Z. Peric, M. Savic, N. Simic, "Design and implementation of nonuniform quantizers for discrete input samples", unpublished (submitted to Elektronika Ir Elektrotechnika). 